\title{
GESTIÓN ACADÉMICA DE LA CARRERA \\ INGENIERÍA AGROFORESTAL
}

\author{
Abner Figueroa Galeano ${ }^{[1]}$ \\ Denis Peralta Zamora ${ }^{[2]}$ \\ Oscar Obregón ${ }^{[3]}$
}

\section{Resumen}

Este estudio se realizó en la URACCAN, Recinto Bilwi, apunta a describir las acciones que ha realizado la coordinación de la carrera de Ingeniería Agroforestal en el primer semestre del 2013. Se ha analizado la gestión académica de esta carrera, se describieron las coordinaciones que se desarrollaron a lo interno entre la carrera y los diferentes estamentos de la Universidad comunitaria e intercultural, con el fin de elaborar una propuesta para mejorar los procesos de gestión. Se desarrolló desde el enfoque del paradigma cualitativo etnometodológico.

Se concluye en realizar un diagnóstico para determinar la demanda de la carrera y actualizar el currículo, ya que este se encuentra desfasado y ante lo cual se han integrado algunos elementos del contexto actual; asimismo, automatizar el Registro Académico y la parte administrativa para agilizar los procesos administrativos y brindar servicios de calidad a la comunidad universitaria.

Palabras clave: Gestión académica; propuesta de mejora; currículum.

\section{Summary}

This study was carried out in URACCAN, Bilwi Campus, and it aims to describe the actions that were made by the coordination of Agroforestry Engineering career in the first semester of the year 2013. An analysis of the academic management of this career was carried out, describing the coordination that was developed internally between careers and the different levels of the community and intercultural University, in order to develop a proposal to improve management processes. The study was developed from a qualitative paradigm with a ethnomethodological approach.

The conclusion of the study is making a diagnosis to determine the demand of the career and update the curriculum since it's out of phase, due to which some elements of the current context have been integrated; but also to automate the academic

\footnotetext{
[1] Master en Desarrollo con identidad, territorialidad, gobernabilidad con mención en manejo de bosque. Coordinador de planificación Recinto Bilwi. afigueroa_44@yahoo.com

[2] Licenciado en Sociología con mención en Autonomía. Coordinador proyecto de Salud Para Todos. denis.peralta1@gmail.com,

[3] Director de Investigación y Post grado Universidad Nacional de Ingeniería. oscardfe@gmail.com
} 
registry and the administrative part to accelerate the administrative processes and provide quality services to the university community.

Keywords: Academic management; proposal for improvement; curriculum.

\section{Introducción}

La carrera de Ingeniería Agroforestal atiende a la comunidad estudiantil universitaria del Recinto Bilwi, desde la fundación de la URACCAN en 1995. Se realizó un diagnóstico curricular en el 2004, con el fin de Identificar las fortalezas y debilidades del currículo vigente (1998) y así fortalecer el presente (2005-2009).

Es el 2013 y todavía no se ha realizado la transformación curricular, hay un déficit de 4 años y aún se trabaja el curriculum (2005-2009). En el 2010 el Consejo Nacional de Universidades (CNU) evalúa la carrera sin embargo es la fecha aún y no se ha recibido el informe de evaluación.

Internamente se coordina con Recursos Humanos para los contratos de docentes, la dirección que asignan las aulas de clase, espacios de oficina y recursos tecnológicos necesarios para llevar a cabo los procesos enseñanza aprendizaje, con registro para ver la cantidad de estudiantes matriculados, biblioteca, institutos y centros de investigación entre otros, es por ellos que se presenta esta propuesta de proyecto con el fin de mejorar todos estos procesos de gestión de la carrera.

\section{Revisión de literatura}

Esta investigación tiene como base referencial las teorías planteadas en relación a la gestión académica.

\section{Gestión}

La gestión se caracteriza por una visión amplia de las posibilidades reales de una organización para resolver alguna situación o para alcanzar un fin determinado. Se define como el conjunto de acciones integradas para el logro de un objetivo a cierto plazo, es la acción principal de la administración y el eslabón intermedio entre la planificación y los objetivos concretos que se pretenden alcanzar (Vázquez 2011 p 55).

Esta referencia de Vásquez, en la vida universitaria está relacionado a la gestión en general del quehacer institucional, para ello se elaboran planes estratégicos de tres a cinco años que guían a las universidades al cumplimiento de sus metas (misión y visión). 


\section{Gestión de la docencia}

En la gestión de la docencia priman tres aspectos fundamentales como lo son la planificación, el monitoreo constante y la evaluación de las acciones de la docencia, para lo cual se requiere de una estructura organizativa funcional eficiente y en esto concuerda con De Miguel (2009) cuando afirma que:

Es un proceso que comprende la planificación, monitoreo y evaluación de las actividades relacionadas con la docencia. Este proceso abarca además el desarrollo de las actividades necesarias para la operación normal de las carreras ofrecidas por la institución. La finalidad fundamental de la gestión de la docencia es garantizar la calidad de la oferta académica. Para esto, debe tener una estructura organizativa y procedimientos eficaces y eficientes (p. 9).

En Nicaragua las Instituciones de Educación Superior se clasifican en públicas, privadas y comunitarias, este hecho denota una gran variedad de determinantes en la gestión de la docencia ya que de acuerdo a su carácter social y filosófico se determinará los aspectos de la gestión a considerarse. Aunque existen muchos aspectos relacionados a la gestión de la docencia se puede preferir por siete rubros esenciales como sugiere el Centro Interuniversitario de Desarrollo (CINDA).

Existe una gran variedad de condicionantes para la gestión docente, dependiendo del tipo de institución, de su tamaño, de su localización, de su carácter público o privado, de la modalidad preferencial para implementar la docencia (presencial, tradicional, no convencional, o no presencial) y del nivel en que se imparte (pregrado o postgrado). Sin embargo en general se pueden establecer siete rubros en la gestión docente: la gestión del currículo, la gestión de asuntos estudiantiles, la gestión del personal docente, la gestión de los recursos materiales y de información, y la planificación y la evaluación global de la función docente. Por cierto se trata de aspectos traslapados e interactivos que sólo se pueden diferenciar para efectos analíticos.

\section{Materiales y métodos}

\section{Paradigma de la investigación}

Cualitativo porque consideramos que la investigación es dinámica, tendrá una riqueza interpretativa, será una experiencia única y los significados se extraerán de los datos. Vamos a explorar y descubrir hasta llegar a una perspectiva general. 


\section{Enfoque de la investigación}

El enfoque de investigación seleccionado es la etnometodología, es una propuesta básica de la sociología que ofrece una perspectiva particular acerca de la naturaleza e indagación del orden social. Se estudia lo que se da por cierto, las prácticas del sentido común a través de las cuales los miembros de la sociedad coordinan, estructuran y entienden sus actividades diarias.

\section{Población y muestra}

La población la comprenden los diferentes estamentos de la Universidad de las Regiones Autónomas de la costa Caribe Nicaragüense que coordinan actividades con la carrera de Ingeniería Agroforestal, siendo estos la Vicerrectoría, la Secretaría Académica, la administración, registro, biblioteca, otras coordinaciones de carrera, los institutos y centros de investigación y la coordinación de Ingeniería Agroforestal.

La muestra corresponde a:

1. Secretaría Académica.

2. Coordinación de Carrera de Ingeniería Agroforestal.

3. La directora del Instituto de Medicina Tradicional y Desarrollo Comunitario.

\section{Método y técnica de recopilación de la información}

Para poder establecer un proceso participativo y consensuado, se trabajó con el método de la entrevista cualitativa y la técnica de entrevista individual. Para complementar la investigación se hizo un análisis de contenido aplicando la técnica de análisis documental (informes de gestión trimestral del recinto Bilwi, actas de consejos técnicos, actas del Consejo Universitario de Recinto) con el fin de interpretar los textos.

\section{Análisis de resultados}

El procesamiento y análisis de datos correspondiente a las entrevistas cualitativas se hizo mediante la triangulación de sujetos creando así categorías de análisis. En el análisis de contenido se hizo lectura cruzada y compartida de los documentos en cuestión, específicamente sobre los hallazgos encontrados relacionado al problema de la investigación de modo que sea posible construir una síntesis e interpretación de los datos. 


\section{Resultados}

\section{Coordinación interna de la carrera Ingeniería Agroforestal}

\section{Estudiantes}

Una vez presentada la documentación a Registro Académico y haberse pre matriculado debe cursar y aprobar las asignaturas del curso propedéutico, siendo estas: Orientación Vocacional, Filosofía Institucional, Matemática y Español, cada una de estas tiene un promedio de 20 puntos.

El primer contacto que tiene la coordinación de Ingeniería Agroforestal con los estudiantes es mediante la presentación de la carrera en el curso propedéutico (visión y misión, perfil de egresado, duración de la carrera, currículum, fotografías y videos de prácticas desarrolladas con estudiantes en proceso de estudio y egresados), posterior a esto el otro momento es la realización de entrevista donde se pretende observar cómo se expresa y desenvuelve el estudiante. La entrevista debe realizarse de forma exigida ya que es parte del puntaje del estudiantes, está la realizan el coordinador con ayuda de los docentes a tiempo completo de la carrera.

Durante el proceso de estudio el coordinador mantiene una estrecha relación con los estudiantes, en cuestiones relacionadas a quejas, elaboración de horarios de exámenes, orientación en cuestiones administrativas que tienen que ver con las normativas del Régimen Académico donde se estipula cuáles son los trámites que se deben realizar para la elaboración de exámenes de suficiencia, exámenes por tutoría, cursos de verano, convalidación de asignaturas, reposición de exámenes, permisos, créditos de asignaturas, prácticas de campo y cuestiones relacionadas al reglamento interno de la Universidad. Además, el coordinador selecciona a los estudiantes en base a criterios de promedio y valores para participar en los diferentes eventos que se realizan a nivel institucional, municipal, regional, nacional e internacional.

\section{Docentes}

El coordinador propone a la Secretaría Académica la contratación de docentes a tiempo completo, la planta docente que trabajará en labores de docencia horaria en el semestre, dirige el plan calendario de docencia y mandata a los docentes a la elaboración del syllabus.

Controla y supervisa la ejecución de las asignaturas y la implementación de las clases prácticas de cada una de ellas según programas y guías que realiza el docente en base al syllabus. Evalúa el proceso educativo desarrollados en las aulas de clase en coordinación con docentes de tiempo completo de otras carreras, concede permiso a los catedráticos por un término no mayor de 3 días, evalúa la actividad docente, 
la autoevaluación docente, solicita la elaboración de contratos al área de Recursos Humanos y pagos a los catedráticos docentes horarios según horas laboradas a la administración, invita a los docentes a las diferentes charlas, capacitaciones, talleres que se desarrollan tanto fuera como dentro del recinto, así mismo les informa acerca de los acuerdos sostenidos en los Consejo Universitario de Recinto (CUR) y Consejos Técnicos y los resultados de los procesos de evaluación docente.

\section{Tecnología}

Se cuenta con la plataforma virtual (Moodle) donde se comparte generalmente con los estudiantes de cuarto y quinto año, como parte de otra metodología de facilitar la docencia. Además, algunas asignaturas por su complejidad utilizan diferentes programas como ArcGIS, infostad, SPSS, AutoCAD, GPS, brújulas, hipsómetros, clinómetros, estereoscopios, centrífuga, microscopios, los diferentes equipos del laboratorio de suelo, agua y biología entre otros. Además de ello se utilizan equipos como data show, internet y computadoras para la realización de las diferentes actividades de la docencia.

Se implementa un sistema para la interacción automatizada de estudiantes y docentes para el proceso de matrícula, consulta de historial académico y registro de calificaciones; sin embargo, esto no ha sido posible ya que el sistema presenta fallas al ingresar y al manipular los datos.

\section{Marco regulatorio vigente}

Para llevar a cabo una administración eficiente en el área de ingeniería, debe cumplir lo establecido en las diferentes normativas de la institución, en primera instancia el estatuto de la URACCAN de donde se desglosa diferentes normativas, reglamentos y políticas institucionales que más hace referencia al quehacer del área:

La mayoría de los docentes desconocen la información que contiene los documentos normativos, es decir, conocen cuales son los nombres de los documentos; pero, desconocen su información, por lo tanto no saben cuál es la orientación hacia el fomento de los valores y la aplicabilidad de estos en caso de violación a la normativa institucional.

\section{Infraestructura}

Se ha destinado una oficina que es utilizada por el coordinador y dos cubículo para los docentes a tiempo completo. Para el ejercicio de la docencia se cuenta con un aula asignada de forma permanente, las otras cuatro son asignadas durante el semestre y estas varían de lugar en base a la cantidad de alumnos. Se tiene un espacio en el recinto donde los estudiantes desarrollan sus prácticas (vivero, gallinero, terreno de siembra). 


\section{Recursos Humanos}

El coordinador cuenta con la colaboración de tres docentes a tiempo completo y un técnico de campo que trabaja como contraparte con la cooperación japonesa (Jica); aún están disponibles dos plazas de docentes ya que uno fue promovido a otro cargo y el otro renunció. Cada semestre se contrata a 9 docentes horarios y se tiene apoyo de 8 personas del personal administrativo y docentes de otras áreas que imparten docencia en la carrera. Se tiene plena coordinación con el área de Recursos Humanos con la elaboración del contrato, deducciones según leyes y carga horaria de los trabajadores a tiempo completo.

\section{Biblioteca}

La biblioteca es el área que cuenta con los medios audiovisuales y documentación. La biblioteca solicita al coordinador la lista de bibliografía a necesitar cuando se cuenta con presupuesto, además el coordinador entrega documentación recibida en los diferentes eventos en que se participa y aquellas investigaciones que se realizan como trabajos de fin de cursos que cumplen con criterios de calidad.Se coordina para la revisión de bibliografías del área antes de iniciar el semestre académico, el coordinador de carrera manda la lista de los docentes que van impartir asignaturas en el semestre para facilitarle los servicio de la biblioteca.

Aunque se cuenta con una gama de material bibliográfico, aún hace falta adquirir más bibliografías para la mayoría de las asignaturas en base al contenido de los programas académicos del currículum de la carrera.

\section{Registro}

El coordinador solicita a registro la lista oficial de los estudiantes matriculados en el semestre, este además lleva control de los créditos de cada estudiante facilitados por los docentes y el coordinador. Este resguarda las actas de calificaciones de los estudiantes. Se tiene coordinación en el registro de exámenes de suficiencia, tutoría, cursos de verano, entre otros. Tanto el coordinador como registro deben de cumplir estrictamente lo que estipula el Régimen Académico.

Se mantiene buena comunicación entre las áreas en actividades como por ejemplo: fotografía para carnet estudiantil, programación de matrícula, oferta académica de cada semestre, listado de egresados al finalizar el año, información de mejores estudiantes de cada semestre, inscripción de temas monográficas, actas de defensa monográfica, cartas de autorización de cursos especiales. El manejo de datos es manual, se auxilia únicamente de hojas dinámicas de Excel, se necesita adaptar el sistema para atender de una forma más eficiente a la comunidad universitaria. 


\section{Recursos financieros}

Al final de cada año se pasa la propuesta presupuestaria a la administración con todos los requerimientos del año siguiente, en el van estipulados todos los gastos de servicios personales, no personales, de materiales e suministros y prácticas. En el Consejo Universitario de la URACCAN (CUU) se aprueba el presupuesto del recinto sin embargo se desconoce el monto aprobado en cada uno de los rubros. Se ha tratado de establecer el sistema administrativo financiero, pero se ha caminado a paso lento, este vendría a ayudar en gran medida y agilizar los trámites administrativos financieros de la institución.

\section{Institutos y Centros de Investigación}

Una de las tareas fundamentales de los institutos y centros de investigación es realizar procesos de extensión social comunitaria y la investigación, es en este quehacer donde participan estudiantes de acuerdo al tipo de investigación. Cuando se involucra a estudiantes y docentes se realizan gestiones con las instancias pertinentes siendo estas la Vicerrectoría, Secretaría Académica y el coordinador de la carrera que es quien garantiza la selección de los mejores estudiantes. Hay poca coordinación entre institutos y la coordinación de Ingeniería Agroforestal por lo cual la integración de estudiantes se da de forma esporádica.

\section{Dirección}

El coordinador responde a Secretaría Académica y al Vicerrector, a él están sujetos los docentes tiempo completo, los docentes horarios y el técnico de campo.

Le corresponde gerenciar el plan curricular de las carreras del área, en coordinación con la Secretaría Académica, Vicerrectoría y Dirección Académica, construye planes operativos anuales, participa en la revisión de diseños curriculares, propone cambios y ajustes de contenido de los programas, dirigir y promover la investigación entre los estudiantes y docentes, dirigir la comisión de carrera del Recinto, es miembro del Consejo Universitario del recinto.

Lo antes mencionado va en correspondencia con lo planteado por De Miguel (2009), todas las actividades que realiza el coordinador están previamente planificadas, para esto se realizan los planes operativos anuales derivados de la planificación estratégica institucional, en ella se enmarcan las actividades relacionadas al cumplimiento de la excelencia académica. 


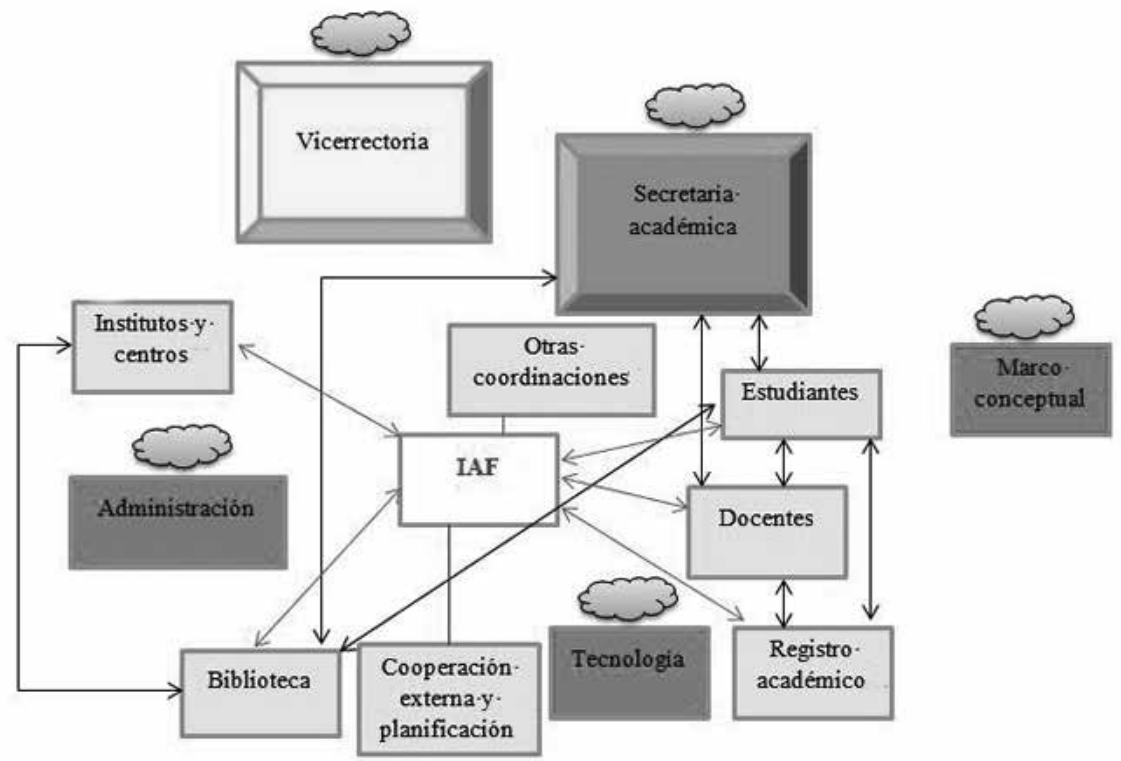

Figura No. 1. Organigrama de coordinación interna de la Carrera Ingeniería Agroforestal .

Fuente: Elaboración propia

\section{Propuesta para mejorar la gestión de la docencia}

\section{Gestión del currículo}

Después de 19 años se necesitar realizar un diagnóstico para identificar las necesidades de la carrera, conocer el nuevo perfil de egreso del ingeniero agroforestal en base a las necesidades del contexto actual y determinar la demanda del mercado social.

\section{Transformación curricular}

El currículo de la carrera lleva 4 años de caducidad, está dirigido a la atención de estudiantes de la modalidad semestral nocturna, desde el 2013 se reciben clases en el turno matutino, al realizar la transformación curricular se debe mantener la pertinencia de este con las costumbres y tradiciones de los pueblos indígenas, mestizos y afrodescendientes.

\section{Gestión estudiantil}

Desde la coordinación no se participa en la selección de los Estudiante, el sistema de selección no está normado, está en proceso de formación, se realiza un curso propedéutico que termina con el examen de admisión donde los 40 promedios más altos; sin embargo, en algunos casos se realizan las acciones afirmativas de beneficio hacia 
una persona que no ha tenido oportunidad, en este caso es enfocado al acceso a la educación, teniendo en cuenta mayor proporción de mujeres, para que encaminen a sus comunidades al desarrollo para alcanzar el buen vivir.

Se debe realizar la selección de estudiantes de otra manera, establecer criterios como el enfoque de género, aspectos de pueblos indígenas, mestizos y afrodescendientes, representatividad étnica, notas de cuarto y quinto año, procedencia y sobre todo tomar en cuenta los valores y conducta presentados en el curso propedéutico.

\section{Sistema de Registro Académico}

Estamos entrando a otra etapa de la vida universitaria en el manejo del sistema estudiantil, ahora se cuenta con mayor cantidad de estudiantes por la apertura de nuevas carreras este año. El informe del primer semestre (2013) refleja que existen 1173 estudiantes matriculados de las cuales 647 son del sexo femenino y 453 del sexo masculino.

Antes lo mencionado el sistema de registro ya no debe hacerse de forma manual, los archivos deben ser sistematizados, la universidad es una entidad que brinda servicios como cartas de egresados, historiales académicos, carta de graduados, certificados, diplomas, entre otros, estos servicios deben ser de calidad. No se puede seguir atrasando los procesos de gestión en el Registro Académico ya que los trámites de algún documento dilatan de 7 a 15 días.

\section{Personal docente}

No existe un criterio para reclutar al personal docente, basado en la experiencia el coordinador hace la oferta académica en las consideraciones tales como profesión, experiencia laboral, estudios investigativos, capacitaciones entre otras, posteriormente se lleva la propuesta a Secretaría Académica y de esta área pasa a Vicerrectoría donde se realizan los cambios.

Lo ideal para la selección de docentes en base a la oferta académica del semestre o anual seria mediante una reunión del coordinador de la carrera, la Secretaría Académica y la Vicerrectoría donde se dialogue y se vean los méritos académicos de los docentes a contratar, esto se debe establecer como una norma y detrás de esta norma establecer el equipo de selección.

\section{Disponibilidad de recursos materiales}

En la parte básica se dispone de aulas, laboratorios de cómputo, biología, química, agua y suelo, la finca académica y sobre todo está el laboratorio natural en las comunidades. 
Se necesita fortalecer los laboratorios, no hay una persona que esté a cargo directamente, se debe contratar a un químico que sea responsable de estos, que vea los reactivos y sus fechas de vencimiento, mantenimiento a los equipos, que lleve el control del uso, que haga cumplir las normas del laboratorio e imparta la docencia.

\section{Venta de servicios}

Se debe potencializar la venta de servicios a las diferentes instituciones, organismos gubernamentales, no gubernamentales, personas naturales y jurídicas para que guíen a las comunidades en la selección de sus áreas de trabajo, fuentes de consumo de agua ideales para la salud y la vida, y en la administración de sus recursos naturales autonómicos con el fin de la búsqueda del desarrollo con identidad para alcanzar el buen vivir, el vivir bien y vivir con dignidad, de acuerdo a las costumbres y tradiciones de cada uno de los pueblos indígenas, mestizos y afrodescendientes de nuestra región y alcanzar calidad en el quehacer de la universidad comunitaria intercultural. La coordinación debe desarrollar licitaciones para adquirir recursos financieros (fondos propios), en el desarrollo del trabajo se debe de involucrar a los estudiantes como parte de su formación profesional según perfil de egreso, brindándole un estipendio como motivación.

\section{Sistema de evaluación}

El sistema evaluativo se realiza de manera cuantitativa o sumativa, en las actualizaciones de los currículum se debe integrar la parte subjetiva o sea la parte formativa que integre los valores

\section{Uno de los entrevistados manifiesta:}

Les digo agarren un papel y pártanlo en cuatro tucos y les digo vos evalúalo a él y dice este me va a evaluar mal, ahhhhhh, yo quiero ver valores también; Tienen 5 años de estar acá y todavía no son amigos, este me va a aplazar, entonces son enemigos esto es una forma de valoración más profunda, el conocimiento ya se los di y sé que tienen conocimiento ya los cuestione con preguntas anteriormente en las clases.

\section{Recursos de información}

La Biblioteca "Fray Gregorio Smutko" cuenta con 34,724 ejemplares de libros, de los cuales no se maneja el dato de bibliografía de áreas afines a la carrera de Ingenieria Agroforestal. Uno de los entrevistados menciona:

La cantidad no es suficiente, pero ese no es el problema, el problemas es no saber utilizar la biblioteca, ni siquiera los libros lo estamos sabiendo utilizar, no es cantidad 
lo que queremos, sino saber utilizar las cosas, pero de que necesitamos si necesitamos de libros, de métodos y sistemas para manejar la información virtual más accesible y asequible para las personas.

Las bibliotecas son un factor a evaluar en el proceso de autoevaluación institucional mandatado por la ley 704 ley creadora del sistema nacional para el aseguramiento de la calidad de la educación y reguladora del Consejo Nacional de Evaluación y Acreditación (CNEA) y del Consejo Nacional de Universidades (CNU), en su artículo 10 numeral 7, menciona disponer de una adecuada biblioteca y recursos de apoyo para las diferentes actividades docentes. Es decir disponer de bibliografía pertinente y suficiente según demanda de los alumnos clasificadas en textos básicos y complementarios de cada asignatura.

\section{Biblioteca virtual}

La Biblioteca de URACCAN pertenece al Sistema Nacional de Bibliotecas Universitarias de Nicaragua (SIBIUN). Se tiene acceso a las Bases de datos en línea ofertadas por HINARI, OARE y AGORA, que ofrecen acceso a universidades de Nicaragua a más de 3,000 títulos de revistas científicas en línea a texto completo.

Como parte del componente del proyecto PERI (Programa para el Fortalecimiento de la información) se cuenta con acceso a más de 10 bases de datos, que se ingresa a través de acceso por IP (clave para acceder a información o conectarse a un dispositivo); en este sentido hace falta promocionar el uso de estas bases de datos, asimismo mantener la capacitación sobre el uso tanto a investigadores, estudiantes de post grados, coordinadores de áreas, docentes y estudiantes de último año de la carrera.

\section{Plan estratégico}

La carrera se integra al Plan Operativo Anual (POA) de la Secretaría Académica, en esa parte no hay una visibilización directa de la carrera. Se está fallando en la práctica de evaluación. Duriez (2009) hace mención que:

al hablar de práctica evaluativa debemos asumir preliminarmente que la evaluación no es un concepto abstracto con existencia aparte de la experiencia humana. Es un constructo social con base en la práctica. Es un proceso reflexivo que emite juicios de valor sobre el estado o situación de un estudiante, docente, programa o institución educativa.

Para saber si se estan desarrollando nuestras actividades de una manera eficiente, se debe adoptar sistemáticamente la práctica de evaluación en la carrera, es la que ayuda a identificar adonde se está fallando y cuáles son las acciones que se debe 
desarrollar para mejorar el quehacer de la carrera y cumplir con las metas del Plan Operativo Anual.

\section{Evaluación del desempeño docente}

Se evalúa de forma occidental, la parte de la filosofía de universidad comunitaria intercultural no se visibiliza, se necesita construir los indicadores, no se puede medir algo que no sabemos y no lo vivimos, el paradigma de universidad comunitaria tiene que ver con el proyecto educativo, su modelo y esto debe estar enfocado a este principio de universidad comunitaria, se debe tener criterios propios porque se trabaja con formatos de otras instituciones o entes reguladores.

Se debe cumplirle al CNU y no solo porque nos da un porcentaje del 6\%, pero sino ante todo por ser una universidad comunitaria intercultural. Uno de los entrevistados menciona: "Dirán que clase de universidad es esa, como van a traer gente de las comunidades a impartir docencia, como los estudiantes se van a ir a vivir allá, cómo van a traer a un anciano a dar clase a una maestría por ejemplo, es un juego allí".

Otro de los entrevistados aduce que: "Hay cosas que no entendemos y falta bastante tiempo para que todos logremos entender, esto no depende del tiempo, depende de que tan claro estén nuestras autoridades y de qué cosa significa eso, si ellos no están claros allí va a ir el caballito, va a seguir el mismo camino, pero si ellos están claros podemos dar el gran salto y hacer el cambio, aunque la gente le tiene miedo al cambio, el cambio es el que provoca desarrollo, te vas a equivocar está bien, pero te reestructuras, tenemos miedo a que nos quiten el $6 \% "$.

Se debe diseñar un sistema de evaluación del desempeño docente pertinente a nuestra realidad, a nuestra razón de ser, con nuestros propios indicadores, con nuestros propios criterios de calidad, donde haya participación de toda la comunidad universitaria que intervienen en los procesos enseñanza aprendizaje, de cómo es el docente con conocimiento occidental o tradicional en relación al docente de una Universidad Comunitaria Intercultural, cómo se mantiene ese equilibrio dentro de las aulas de clase.

\section{Los docentes de la URACCAN no deben ser como Navarro menciona en su artículo}

El profesor aceptaba a los estudiantes que consideraba suficientemente preparados "y en ellos" no había profesores que leían en cátedra los libros de texto, ni alumnos que pasivamente los escuchaban, sino compañeros que trabajaban en común en laboratorios, bibliotecas y en investigación. Como se sabe, el modelo de seminarios de nuestras universidades, tienen más un enfoque clerical, dado que los profesores más parecen sacerdotes predicando la palabra de Dios, que un ejercicio colectivo de afirmación del saber y construcción del conocimiento. 


\section{Conclusiones}

De manera general se percibe que existe coordinación entre la carrera de Ingeniería Agroforestal y los diferentes estamentos de la universidad, claro está que las coordinaciones son diferentes en cada una de las áreas. Existe coordinación constante con la Vicerrectoría, la Secretaría Académica, la Administración en aspectos relacionados a la tecnología y cumplimiento del marco legal; con los institutos y centros de investigación, Registro Académico cooperación externa, planificación, biblioteca, estudiantes, con los docentes las coordinaciones se realizan de manera más aislada cuando la situación lo amerita.

Es necesaria la actualización del currículum de la carrera de Ingeniería Agroforestal, ya lleva cuatro años de desfase y se encuentra en un nuevo contexto donde hay otras necesidades. La coordinación de Ingeniería agroforestal debe potencializar la venta de servicios para así mantener sus fondos propios y suplir las necesidades básicas de la carrera.

Las evaluaciones parciales deben integrar aspectos relacionados a valores y la filosofía de la institución. Se debe de establecer sistemas computarizados en el área de Registro Académico y la administración para el manejo de las notas y el pago de aranceles.

\section{Lista de referencia}

CNEA Ley 704. (2009). Ley Creadora del Sistema Nacional para el Aseguramiento de la Calidad de la Educación y Reguladora del Consejo Nacional de Evaluación y Acreditación. Publicada en Managua el lunes 12 de septiembre del 2011 en la Gaceta numero 172.

Centro Interuniversitario de Desarrollo (S/f, CINDA). Gestión Docente Universitaria. Modelos comparados.

De Miguel, José Agustín (2009). La Gestión Docente. UCATECI.

Duriez, Maribel (2099). Cita Textual de Capítulo III- Tesis Doctoral: La cultura organizacional y su influencia en la práctica evaluativa. El caso de la Universidad Nacional de Ingeniería. Publicado en Universidad de Costa Rica

Evangelina Vázquez Herrera (2001). Modelo de gestión Educativa Estratégica. Programa Escuela de Calidad. México Pág. 55. 
Navarro, Mejía David (S/f). La construcción de un modelo posible de Universidad. Articulo otorgado por la PHD, Maribel Duriez en la especialización en Gestión Universitaria 2012-2013.

URACCAN. (2013). Informe de gestión II trimestre. Bilwi. Puerto Cabezas

URACCAN (2004). Diseño curricular de la carrera de Ingeniería Agroforestal. Diciembre Pág. 6 